\title{
Dispersion polymerization of anionic polyacrylamide in an aqueous salt medium
}

\author{
Lu Jiao, Peng Bo*, Li Mingyuan, Lin Meiqin and Dong Zhaoxia \\ Enhanced Oil Recovery Research Center, China University of Petroleum, Beijing 102249, China \\ (C) China University of Petroleum (Beijing) and Springer-Verlag Berlin Heidelberg 2010
}

\begin{abstract}
Anionic polyacrylamide dispersions were prepared by dispersion polymerization in an aqueous salt medium, using acrylamide(AM) and acrylic acid(AA) as monomers and anionic polyelectrolytes as stabilizer. Effects of salt concentration, and molecular weight and concentration of stabilizers on the stability of the dispersions were investigated using a HAAKE rheometer and optical microscopy. The results showed that stable anionic polyacrylamide dispersions, consisting of smooth, spherical, polydisperse particles, could be obtained under the conditions of salt concentration ranging from $26 \mathrm{wt} \%$ to $30 \mathrm{wt} \%$, concentration of stabilizers from $1.2 \mathrm{wt} \%$ to $1.8 \mathrm{wt} \%$, and intrinsic viscosity of stabilizers from $2.98 \mathrm{dL} \cdot \mathrm{g}^{-1}$ to $3.74 \mathrm{dL} \cdot \mathrm{g}^{-1}$. The apparent viscosity of the stable dispersions changed very little with the shear rate, showing Newton fluid behavior.
\end{abstract}

Key words: Dispersion polymerization, aqueous salt medium, stability, micromorphology, apparent viscosity

\section{Introduction}

Polyacrylamide (PAM) is an important water-soluble polymer. It can be used as a flocculant for water disposal, strength additives for paper manufacture, oil displacement agents for enhanced oil recovery, and so on (Yan, 1998).

At present, anionic polyacrylamide is synthesized mainly by two methods, namely solution polymerization and inverse emulsion/microemulsion polymerization. Solution polymerization has been adopted commonly, but the dissolution process of the dry powder is often tedious. When dissolution of PAM is required, inverse microemulsion/ emulsion polymerization is chosen. This uses hydrocarbon as the continuous phase and various surfactants as emulsifiers. The main advantage of this method is that it can obtain highmolecular weight anionic polyacrylamide and achieve high conversion rates during polymerization, and the anionic polyacrylamide has good solubility. But it will result in secondary pollution to the environment as it has organic solvents and emulsifiers added in the reaction system.

Dispersion polymerization can be defined as a special precipitation polymerization in which polymerization is carried out in the presence of a suitable stabilizer (Fang et al, 2006). Monomer, stabilizer, and initiator are dissolved in the aqueous medium before polymerization, and the prereaction system is a homogeneous solution. PAM polymer is precipitated from the aqueous medium due to its low solubility when the formed polymer chains reach critical

*Corresponding author. email: cbopeng@cup.edu.cn

Received November 18, 2009 chain length during polymerization. The precipitated polymers form spherical micro-particles through aggregation and the particles are suspended in the medium by the adsorption of stabilizer. There are several advantages of dispersion polymerization, such as no secondary pollution, high solid content and rapid dissolution (Fang et al, 2006). Until now, most of the papers on dispersion polymerization of hydrophilic monomer acrylamide mainly focus on an alcohol-water medium (Ray and Mandal, 1997; Ray and Mandal, 1999; Ye et al, 2002; Guha and Mandal, 2004; Han et al, 2004; Cui et al, 2002; Wang et al, 2005), and a few papers focus on dispersion polymerization of acrylamide or of acrylamide with cationic monomer in an aqueous salt medium (Cho et al, 2002; Song et al, 2003; Ye et al, 2003; Chen et al, 2006a; 2006b; Guo et al, 2007). Only a few papers focus on dispersion polymerization of acrylamide and anionic monomer acrylic acid (Liu et al, 2006; Liu et al, 2007).

In this paper, anionic polyacrylamide dispersions are synthesized by the dispersion polymerization method with acrylamide as primary feedstock and acrylic acid as secondary feedstock in a salt medium .

The effects of salt concentration, molecular weight and concentration of stabilizers on the stability of anionic polyacrylamide dispersions were investigated by measurements of the apparent viscosity and the stability of the anionic polyacrylamide dispersions and micromorphology observations.

\section{Experimental}

\subsection{Materials}

Reagents used were: Acrylamide (AM) and ammonium 
persulfate, A.R. Grade, supplied by Guangdong Shantou Xilong Chemical Co., Ltd; Acrylic acid (AA) and sodium bisulfite, A.R. Grade, Beijing Yili Fine Chemicals Co., Ltd; Sodium hydroxide, A.R. Grade, Beijing Modern Eastern Fine Chemicals Co., Ltd. Stabilizers with the same structure but different molecular weights were prepared in the laboratory, and the intrinsic viscosity $([\eta])$ was determined in a 0.5 $\mathrm{mol} / \mathrm{L}(\mathrm{NaCl})$ aqueous solution with an Ubbelhode capillary viscometer at $25.0{ }^{\circ} \mathrm{C}$; Other reagents were all A.R. Grade and used as received.

\subsection{Preparation and characterization}

Acrylamide, acrylic acid, sodium hydroxide, salts, stabilizer, and deionized water were added to a $500 \mathrm{~mL}$ fournecked flask equipped with an anchor-like paddle stirrer, a reflux condenser, and a nitrogen inlet. After purging with nitrogen for half an hour at $30{ }^{\circ} \mathrm{C}$ in a water bath, polymerization was initiated by injecting the water-soluble initiator ammonium persulfate (APS) and $\mathrm{NaHSO}_{3}$ into the stirred reaction system. The polymerization was allowed to proceed for $22 \mathrm{~h}$ and then the anionic polyacrylamide dispersions were obtained. The apparent viscosity of the dispersions was measured at $25{ }^{\circ} \mathrm{C}$ with a HAAKE RS 600 Rheometer (Thermo Haake Co., Ltd., Germany). The micromorphology of the anionic polyacrylamide dispersions was observed using a XS-18 (304) biological photomicroscope (Nanjing Jiangnan Photoelectric Co., Ltd., China).

\section{Results and discussion}

\subsection{Effect of salt concentration}

The concentration of salt has a strong effect on the stability of the dispersions. If the concentration is too low, the anionic polyacrylamide particles does not salt out easily from the medium, and solution polymerization takes place. If the concentration is too high, the anionic polyacrylamide dispersions have lower thermodynamic stability, and it is difficult to obtain stable dispersions.

Fig. 1 shows the relationship between the apparent viscosity and shear rate of the anionic polyacrylamide dispersions in an aqueous medium with different salt concentrations. When the salt concentration was low (20$24 \mathrm{wt} \%$ ), the apparent viscosity of the dispersions decreased gradually with increasing shear rate. At the same shear rate, the higher the salt concentration, the lower the apparent viscosity of the dispersions. At high salt concentration (26$30 \mathrm{wt} \%$ ), the apparent viscosity of the dispersions was almost unchanged with increasing shear rate, and at the same shear rate, the apparent viscosity changed very little as the salt concentration changed from $26 \mathrm{wt} \%$ to $30 \mathrm{wt} \%$.

The stability of the anionic polyacrylamide dispersions is shown in Table 1 . When the salt concentration was too low $(20-24 \mathrm{wt} \%)$, the dispersions were not stable, and would separate into layers within 10 hours. At high salt concentration (26-30 wt\%), stable anionic polyacrylamide latex dispersions were obtained. Fig. 2 shows the photographs of the stable (a) and unstable (b) dispersions. In the unstable dispersion, the interface between particles and aqueous medium can not be distinguished. In the stable dispersion, there are amounts of smooth-surfaced, spherical, polydisperse particles.

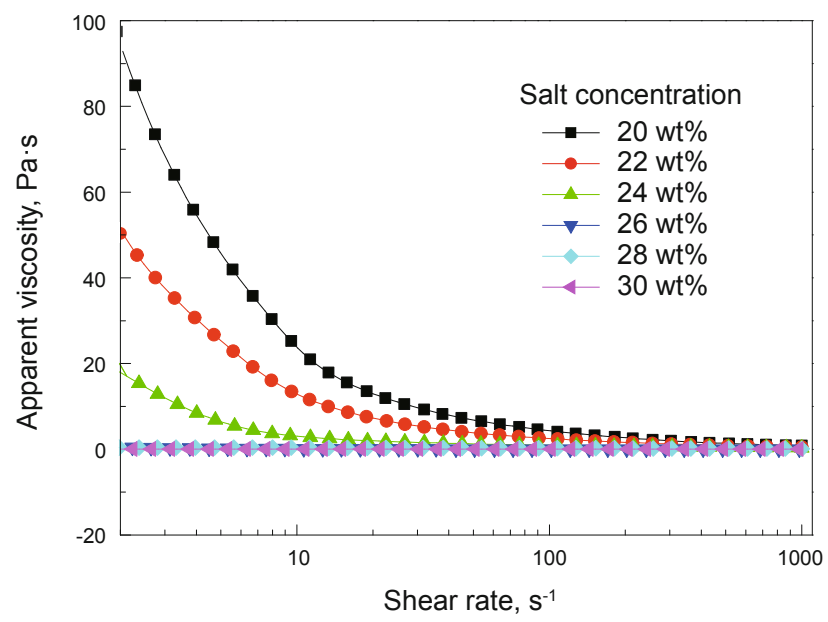

Fig. 1 Apparent viscosity of the anionic polyacrylamide dispersions as a function of shear rate in aqueous medium with different salt concentrations

Conditions: monomer, $15 \mathrm{wt} \%$; molar ratio of $\mathrm{AM} / \mathrm{AA}, 7: 3$; AA, neutralized by $\mathrm{NaOH}$ solution and neutralization degree reached $15 \%$; intrinsic viscosity $[\eta]$ of stabilizer, $3.74 \mathrm{dL} \cdot \mathrm{g}^{-1}$; stabilizer, $1.5 \mathrm{wt} \%$; initiator $0.01 \mathrm{wt} \%$

Table 1 Stability of anionic polyacrylamide dispersions

\begin{tabular}{|c|c|c|c|c|c|c|}
\hline $\begin{array}{c}\text { Salt } \\
\text { concentration, } \\
\text { wt } \% \\
\end{array}$ & 20 & 22 & 24 & 26 & 28 & 30 \\
\hline $\begin{array}{l}\text { Stability of } \\
\text { dispersions }\end{array}$ & Unstable & Unstable & Unstable & Stable & Stable & Stable \\
\hline
\end{tabular}

Conditions: monomer, $15 \mathrm{wt} \%$; molar ratio of $\mathrm{AM} / \mathrm{AA}, 7: 3$; neutralization degree, $15 \%$; Intrinsic viscosity [ $\eta$ ] of stabilizer, 3.74 $\mathrm{dL} \cdot \mathrm{g}^{-1}$; stabilizer, $1.5 \mathrm{wt} \%$; initiator $0.01 \mathrm{wt} \%$

When the concentration of salt was relatively low (20-24 $\mathrm{wt} \%$ ), part of the polymer salted out from the salt solution, and some polymer remained in the salt solution. A network structure formed by tangled polymer molecule chains, leading to high apparent viscosity and undistinguished micromorphology of the particles, hence the dispersion system was not stable. An increasing shear rate can destroy the network structure, so the system shows shear thinning. When the salt concentration was increased to a high value (26-30 wt\%), the polymer particles would salt out entirely from the aqueous medium, forming a stable dispersion because the stabilizer absorbed on the spherical particles and dispersed them. In this case, the apparent viscosity of the dispersions is very low, and almost unchanged with the shear rate. This result agrees well with that reported in literature (Zhou et al, 1987), namely, the more regular the disperse phase, the smaller the apparent viscosity and the more stable the dispersions. 

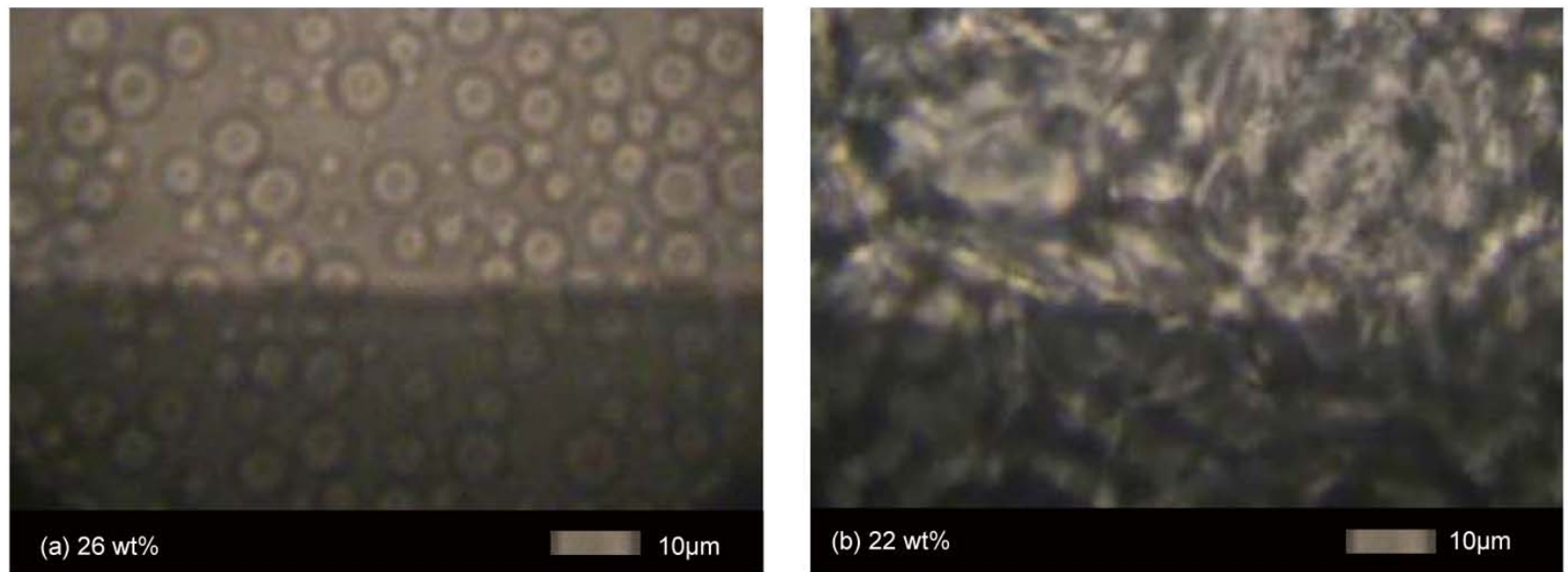

Fig. 2 Effect of salt concentration on the micromorphology of the anionic polyacrylamide dispersion Polymerization condition: monomer, $15 \mathrm{wt} \%$; molar ratio of AM/AA, 7:3; neutralization degree, $15 \%$; Intrinsic viscosity [ $\eta$ ] of stabilizer, 3.74 $\mathrm{dL} \cdot \mathrm{g}^{-1}$; stabilizer, $1.5 \mathrm{wt} \%$; initiator, $0.01 \mathrm{wt} \%$; salt, $26 \mathrm{wt} \%$ (a), $22 \mathrm{wt} \%(\mathrm{~b})$

\subsection{Effect of stabilizer concentration}

Fig. 3 shows the relationship between the apparent viscosity of the anionic polyacrylamide dispersions, prepared with different concentrations of stabilizer, and the shear rate. When the stabilizer concentration was in the range of $0.6-1.8$ $\mathrm{wt} \%$, the apparent viscosity of the dispersions changed little with the shear rate. But when the stabilizer concentration was $2.4 \mathrm{wt} \%$, the apparent viscosity decreased greatly with the shear rate. Furthermore, at the same shear rate, the apparent viscosity increased gradually with stabilizer concentration in the range of 0.6-1.8 wt \%, while it increased greatly when the stabilizer concentration was increased to $2.4 \mathrm{wt} \%$.

Table 2 shows the stability of the anionic polyacrylamide

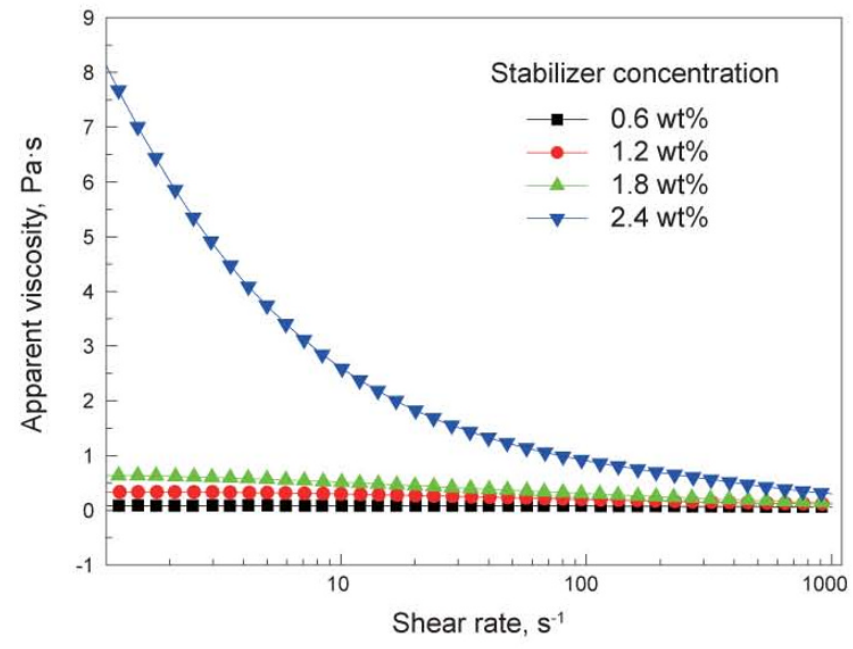

Fig. 3 Effect of stabilizer concentration on the apparent viscosity of the anionic polyacrylamide dispersions

Conditions: monomer, $15 \mathrm{wt} \%$; molar ratio of $\mathrm{AM} / \mathrm{AA}, 7: 3$; neutralization degree, $15 \%$; salt, $26 \mathrm{wt} \%$; intrinsic viscosity [ $\eta$ ] of stabilizer, $3.52 \mathrm{dL} \cdot \mathrm{g}^{-1}$; initiator, $0.01 \mathrm{wt} \%$ dispersions prepared with different stabilizer concentrations. It can be seen that the dispersions were not stable when the concentration of stabilizer was too low $(0.6 \mathrm{wt} \%)$ or too high $(2.4 \mathrm{wt} \%)$. The dispersions were stable when the stabilizer concentration ranged from $1.2 \mathrm{wt} \%$ to $1.8 \mathrm{wt} \%$.

Fig 4 shows the micromorphology of the stable (a) and unstable (b) dispersions. Smooth spherical polydisperse particles exist in the stable dispersion, while the particles in the unstable dispersion were chain shaped.

Table 2 Stability of anionic polyacrylamide dispersions

\begin{tabular}{ccccc}
\hline $\begin{array}{c}\text { Concentration of } \\
\text { stabilizer, } \mathrm{w} \%\end{array}$ & 0.6 & 1.2 & 1.8 & 2.4 \\
\hline Stability of dispersions & Unstable & Stable & Stable & Unstable \\
\hline
\end{tabular}

Conditions: monomer, $15 \mathrm{wt} \%$; molar ratio of AM/AA, 7:3; neutralization degree, $15 \%$; salt, $26 \mathrm{wt} \%$; Intrinsic viscosity $[\eta]$ of stabilizer, $3.52 \mathrm{dL} \cdot \mathrm{g}^{-1}$; initiator, $0.01 \mathrm{wt} \%$

When the concentration of stabilizer was $0.6 \mathrm{wt} \%$, the stabilizer was not concentrated enough to stabilize all the particles salted out from the aqueous medium, and the particles easily stuck to one another. Hence the dispersion was unstable, forming a weak gel during the standing time. When the stabilizer concentration was $2.4 \mathrm{wt} \%$, the extra stabilizer played a role in bridging between the particles, forming bead-shaped chains (Fig.4 (b)), and the dispersion was also not stable. In these two cases, the apparent viscosity of the dispersions is high, and the dispersions showed shear thinning. As the molecular weight of the product, prepared with a stabilizer concentration of $0.6 \mathrm{wt} \%$, was very low $\left([\eta]=1.19 \mathrm{dL} \cdot \mathrm{g}^{-1}\right)$, the gelation was not obvious and the apparent viscosity was low. With a stabilizer concentration in the range of $1.2-1.8 \mathrm{wt} \%$, the stabilizer concentration and the salted-out polymer particles matched quite well and stable dispersions with spherical particles were obtained. 

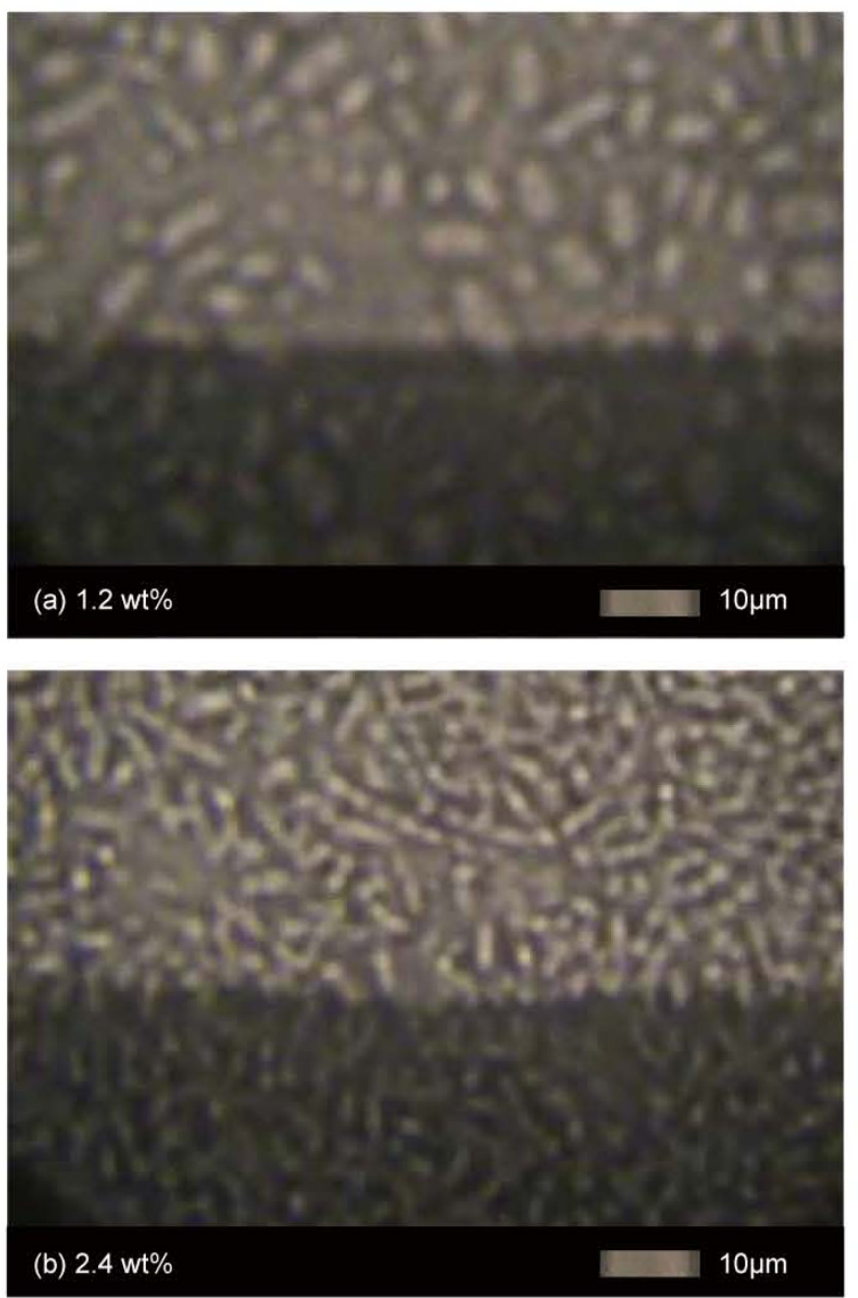

Fig. 4 Effect of stabilizer concentration on the micromorphology of anionic polyacrylamide dispersions

Condition: monomer, $15 \mathrm{wt} \%$; molar ratio of AM/AA, 7:3; neutralization degree, $15 \%$; salt, $26 \mathrm{wt} \%$; Intrinsic viscosity [ $\eta$ ] of stabilizer, 3.52 $\mathrm{dL} \cdot \mathrm{g}^{-1}$; initiator, $0.01 \mathrm{wt} \%$; stabilizer, $1.2 \mathrm{wt} \%$ (a), $2.4 \mathrm{wt} \%(\mathrm{~b})$

\subsection{Effect of stabilizer molecular weight}

Fig. 5 shows the relationship between the apparent viscosity of the anionic polyacrylamide dispersions and the shear rate. When the intrinsic viscosity of stabilizer was too high $\left([\eta]=5.62 \mathrm{dL} \cdot \mathrm{g}^{-1}\right)$ or too $\operatorname{low}\left([\eta]=2.04 \mathrm{dL} \cdot \mathrm{g}^{-1}\right)$, the apparent viscosity decreased greatly with the shear rate. When the intrinsic viscosity of stabilizer was in the range of 2.98-3.74 $\mathrm{dL} \cdot \mathrm{g}^{-1}$, the apparent viscosity changed very little with the shear rate.

Table 3 shows the stability of the anionic polyacrylamide dispersions prepared by using stabilizers with different molecular weights. The dispersions were not stable when the intrinsic viscosity of the stabilizer was too high or too low. When the intrinsic viscosity of the stabilizer was in the range of $2.98-3.74 \mathrm{dL} \cdot \mathrm{g}^{-1}$, the dispersions were stable.

Fig 6 shows the micromorphology of the stable (a) and unstable (b) dispersions. It can be seen that irregular blocks exist in the unstable dispersion, while there are amounts of smooth surfaced, spherical, polydisperse particles in the stable dispersion.

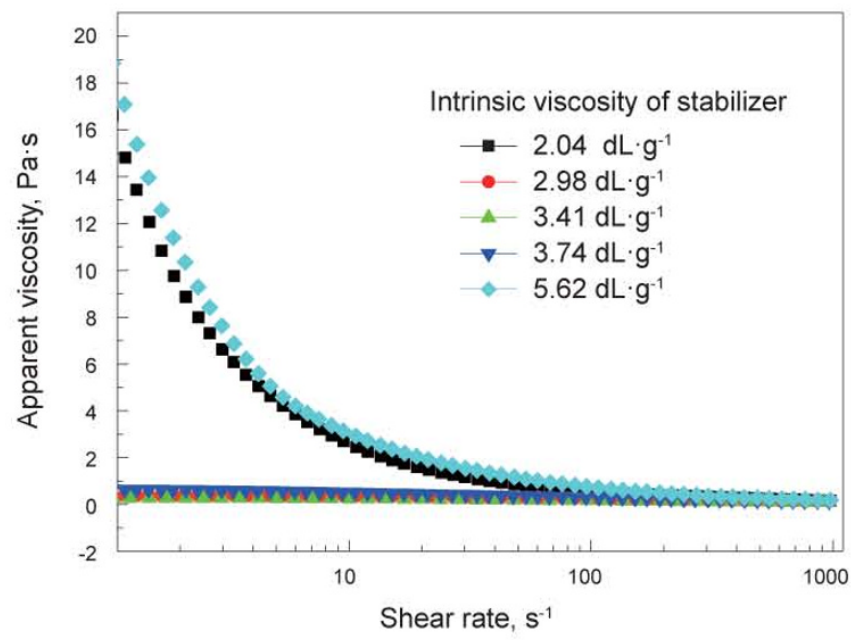

Fig. 5 Effects of intrinsic viscosity of stabilizer on the apparent viscosity of anionic polyacrylamide dispersions

Conditions: monomer $15 \mathrm{wt} \%$, molar ratio of AM/AA, 7:3, neutralization degree $15 \%$, salt $26 \mathrm{wt} \%$, stabilizer $10 \mathrm{wt} \%$, initiator $0.01 \mathrm{wt} \%$
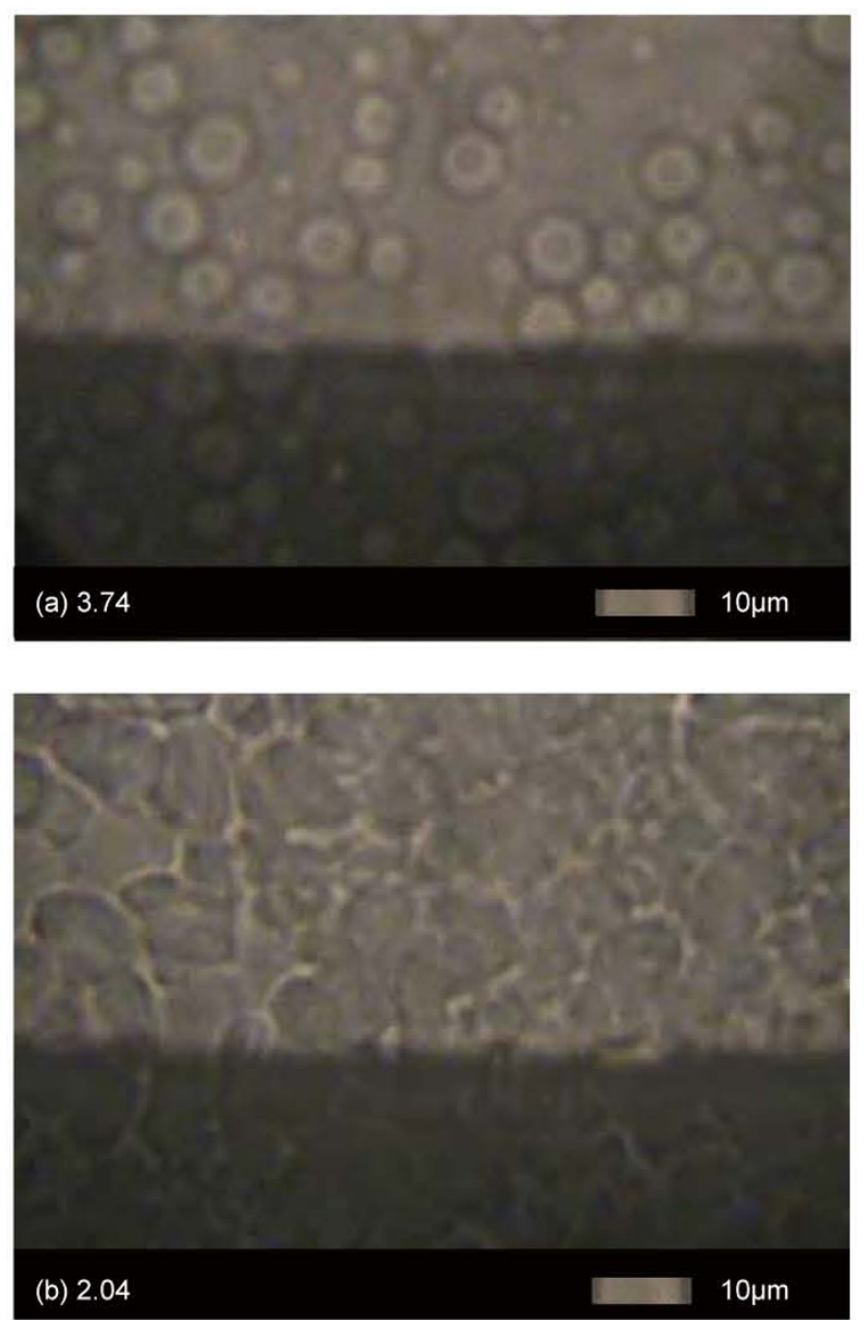

Fig. 6 Effect of molecular weight of stabilizer on the micromorphology of anionic polyacrylamide dispersion

Conditions: monomer, $15 \mathrm{wt} \%$; molar ratio of AM/AA, 7:3; neutralization degree, $15 \%$; salt, $26 \mathrm{wt} \%$; stabilizer, $10 \mathrm{wt} \%$; initiator, $0.01 \mathrm{wt} \%$; Intrinsic viscosity $[\eta]$ of stabilizer, $2.04 \mathrm{dL} \cdot \mathrm{g}^{-1}(\mathrm{a}), 3.74 \mathrm{dL} \cdot \mathrm{g}^{-1}$ (b) 
Table 3 Stability of anionic polyacrylamide dispersions

\begin{tabular}{|c|c|c|c|c|c|c|c|}
\hline $\begin{array}{c}\text { Stabilizer }[\eta] \text {, } \\
\mathrm{dL} \cdot \mathrm{g}^{-1}\end{array}$ & 2.04 & 2.51 & 2.98 & 3.41 & 3.74 & 5.62 & 6.28 \\
\hline $\begin{array}{c}\text { Stability of } \\
\text { dispersions }\end{array}$ & Unstable & Unstable & Stable & Stable & Stable & Unstable & Unstable \\
\hline
\end{tabular}

Conditions: monomer, $15 \mathrm{wt} \%$; molar ratio of $\mathrm{AM} / \mathrm{AA}, 7: 3$; neutralization degree, $15 \%$; salt, $26 \mathrm{wt} \%$; stabilizer, $10 \mathrm{wt} \%$; initiator $0.01 \mathrm{wt} \%$

When the intrinsic viscosity of the stabilizer was too low $\left([\eta]=2.04 \mathrm{dL} \cdot \mathrm{g}^{-1}\right)$, the short molecular chains could not inhibit aggregation between the particles through steric hindrance and electrostatic repulsion. The particles could easily stick to each other, forming irregular blocks. So the anionic polyacrylamide dispersion was unstable. Furthermore, the apparent viscosity of the dispersion was very high, and showed shear thinning behavior. When the intrinsic viscosity of the stabilizer was too high $\left([\eta]=5.62 \mathrm{dL} \cdot \mathrm{g}^{-1}\right)$, the long molecular chains could be bridged between the particles and the anionic polyacrylamide dispersion was also not stable. The stabilizer itself also contributed a lot to the high apparent viscosity of the dispersion. When the stabilizer with suitable intrinsic viscosity was used $\left([\eta]=2.98-3.74 \mathrm{dL} \cdot \mathrm{g}^{-1}\right)$, stabilization efficiency was the highest, and the anionic polyacrylamide dispersions contained smooth spherical particles. The dispersions behaved as a Newton fluid and were stable .

Dispersion polymerization systems consists of monomer, dispersion medium, stabilizer, and initiator. During dispersion polymerization, the stabilizer combines with the salted-out polymer particles, and then the dispersion is stabilized through steric hindrance and charge repulsion. The stabilization efficiency of a stabilizer depends on its state in the medium, the length of its molecules, and its amount, so the concentration of aqueous salt solution, molecular weight and concentration of the stabilizer are of great importance in preparing stable dispersions.

The molecular weight and concentration of the stabilizer directly affect the stabilization efficiency. When the concentration or intrinsic viscosity of the stabilizer is too high, the extra amount of stabilizer or the extra-long molecular chains can bridge between the polymer particles, leading to an unstable dispersion, as shown in Fig. 4(b). When the concentration or the molecular weight is too low, the amount of stabilizer or the length of the molecular chains is not enough to stabilize the dispersion system, and aggregation could happen between the particles (Fig. 6(b)), forming weak gel. In the two cases, the apparent viscosity is very high, and decreases greatly with the shear rate. Therefore, the molecular weight and concentration of stabilizer should be selected and optimized. In this paper, the optimum range for the intrinsic viscosity of stabilizer is $2.98-3.74 \mathrm{dL} \cdot \mathrm{g}^{-1}$, in concentrations between 1.2-1.8 wt \%.

The extensibility of the stabilizer molecular chains is affected by the concentration of salt in the aqueous medium. The higher the salt concentration, the less the extensibility of the stabilizer molecular chains. Meanwhile, the salt concentration affects the solubility of the formed polymers in the aqueous medium. The lower the salt concentration, the higher the molecular weight of the polymers which will be salted out and the smaller the amount of the salted-out polymer. So it is necessary to determine the suitable range of salt concentrations for the balance between the expansion extent of stabilizer molecular chains and the efficient salting out of the formed polymer to prepare stable dispersions. The satisfactory salt concentration in this study is in the range of 26-30 wt $\%$.

\section{Conclusion}

1) Stable anionic polyacrylamide dispersions could be prepared under the conditions of salt concentration ranging from $26 \mathrm{wt} \%$ to $30 \mathrm{wt} \%$, concentration of stabilizers from 1.2 $\mathrm{wt} \%$ to $1.8 \mathrm{wt} \%$, and intrinsic viscosity of stabilizers from 2.98 $\mathrm{dL} \cdot \mathrm{g}^{-1}$ to $3.74 \mathrm{dL} \cdot \mathrm{g}^{-1}$.

2) The apparent viscosity of the stable dispersions was almost unchanged with shear rate, and they behaved as Newtonian fluids.

3) The stable dispersions contained smooth-faced, spherical, polydisperse particles.

\section{References}

Chen D N, Liu X G, Yue Y M, et al. Dispersion copolymerization of acrylamide and cationic monomer in aqueous solution of ammonium sulfate. Acta Polymerica Sinica. 2006a. (9): 1074-1077 (in Chinese)

Chen D N, Liu X G, Yue Y M, et al. Dispersion copolymerization of acrylamide with quaternary ammonium cationic monomer in aqueous salt solution. European Polymer Journal. 2006b. 42: 12841297

Cho M S, Yoon K J and Song B K. Dispersion polymerization of acrylamide in aqueous solution of ammonium sulfate: synthesis and characterization. J Appl Polym Sci. 2002. 83: 1397-1405

Cui Y, Zhang S X and Yang J H. Synthesis of high molecular weight polyacrylamide by suspension polymerization. Journal of Jinan University (Sci. \& Tech.). 2002. 16(4): 327-328 (in Chinese)

Fang D B, Guo R W, Ha R H, et al. Polymers of Acrylamide. Beijing: Chemical Industry Press. 2006. 270 (in Chinese)

Guha S and Mandal B.M. Dispersion polymerization of acrylamide III. Partial isopropyl ester of poly(vinyl methyl ether-alt-maleic anhydride) as a stabilizer. J Colloid Interface Sci. 2004. 271(1): 5559

Guo R W, Zhao Z F and Jin Q M. Dispersion copolymerization of acrylamide/cationic monomer in aqueous salt solution. Chemical Industry and Engineering. 2007. 24(2): 129-133 (in Chinese)

Han L, Ning R C and Xie G. Preparation of a water-in-water emulsion of polyacrylamide by dispersion polymerization. Journal of Functional Polymers. 2004. 17(3): 493-495 (in Chinese)

Liu X G, Chen D N and Zhang W D. Dispersion polymerization of acrylic acid in aqueous media. Chemical Journal of Chinese Universities. 2006. 27(2): 394-396 (in Chinese)

Liu X G, Xiang S, Yue Y M, et al. Preparation of poly(acrylamide-coacrylic acid) aqueous latex dispersions using anionic polyelectrolyte as stabilizer. Colloids Surf. A: Physicochem. Eng. Aspects. 2007. doi: 10.1016/j. colsurfa.2007.06. 007

Ray B and Mandal B M. Dispersion polymerization of acrylamide. Langmuir. 1997. 13: 2191-2196

Ray B and Mandal B M. Dispersion polymerization of acrylamide: 
Part II. 2, 2'- Azobisisobutyronitrile Initiator. J. Polym. Sci. Polym. Chem. 1999. 37: 493-499

Song B K, Cho M S, Yoon K J, et al. Dispersion polymerization of acrylamide with quaternary ammonium cationic comonomer in aqueous solution. J. Appl. Polym. Sci. 2003. 87: 1101-1108

Wang W, Liu L Y, Huang Z H, et al. Study of photoinitiated dispersion polymerization of acrylamide by using graft copolymer of acrylic acid and nonylphenoxypoly(ethylene oxide) macromonomers as dispersant. Acta Polymerica Sinica. 2005. (3): 320-326 (in Chinese)

Yan R X. Water Soluble Polymers. Beijing: Chemical Industry Press. 1998 (in Chinese)
Ye Q, Zhang Z C and Ge X W. Highly efficient flocculant synthesized through the dispersion copolymerization of water-soluble monomers induced by $\gamma$-ray irradiation: synthesis and polymerization kinetics. J. Appl. Polym. Sci. 2003. 89: 2108-2115

Ye Q, Zhang Z C, Jia H T, et al. Formation of monodisperse polyacrylamide particles by radiation-induced dispersion polymerization: Particle size and size distribution. Journal of Colloid and Interface Science. 2002. 253: 279-284

Zhou Z K, Gu T R and Ma J M. Colloid Chemistry. Beijing: Peking University Press. 1987 (in Chinese)

(Edited by Zhu Xiuqin) 